\title{
On 2-factors with long cycles in cubic graphs*
}

\author{
André Kündgen ${ }^{\dagger}$ \\ Department of Mathematics, California State University San Marcos \\ San Marcos, California 92096, USA \\ R. Bruce Richter \\ Department of Combinatorics \& Optimization, University of Waterloo \\ Waterloo, Ontario, N2L 3G1, Canada
}

Received 25 May 2010, accepted 6 October 2010, published online 23 March 2011

\begin{abstract}
Every 2-connected cubic graph $G$ has a 2-factor, and much effort has gone into studying conditions that guarantee $G$ to be Hamiltonian. We show that if $G$ is not Hamiltonian, then $G$ is either the Petersen graph or contains a 2 -factor with a cycle of length at least 7 . We also give infinite families of, respectively, 2- and 3-connected cubic graphs in which every 2-factor consists of cycles of length at most, respectively, 10 and 16.
\end{abstract}

Keywords: Cubic graph, 2-factor, long cycle, snark, infinite graph

Math. Subj. Class.: 05C38, 05C45, 05C70

\section{Introduction}

A theorem of Petersen [6] states that every cubic 2-connected graph $G$ has a perfect matching, and thus a 2-factor. Much effort has gone into studying conditions for when $G$ has a 2 -factor consisting of only one cycle, that is $G$ is Hamiltonian. This motivates our definition of $\ell(G)$ as the length of a longest cycle in any 2-factor of $G$, and any such cycle as a longest 2-factor cycle. Obviously $G$ is Hamiltonian precisely when $\ell(G)=|V(G)|$. We also call any 2-factor achieving $\ell(G)$ a longest 2 -factor of $G$. (All graphs in this work are simple; that is, there are no loops or multiple edges.)

Petersen's theorem says that $\ell(G)$ is well-defined when $G$ is cubic and 2-connected. A typical proof of Petersen's theorem simply verifies that Tutte's 1-factor condition holds for

\footnotetext{
* Dedicated to Michael O. Albertson.

${ }^{\dagger}$ Corresponding author.

E-mail addresses: akundgen@csusm.edu (André Kündgen), brichter@math.uwaterloo.ca (R. Bruce Richter)
} 
cubic 2-connected graphs. Schönberger [7] (see also [9, Exercise 3.3.17] and [2, Corollary 4.4]) found a strengthening of Petersen's theorem by showing that every edge $f$ in a 2-connected cubic graph $G$ can be extended to a 1-factor, and so $G$ contains a 2-factor avoiding $f$. This also guarantees that $G$ must have a 2-factor using any two specified edges $e_{1}, e_{2}$, since we can subdivide $e_{1}, e_{2}$ and connect the new vertices with the new edge $f$ and then consider the 2-factor avoiding $f$ in the new cubic graph.

Schönberger's theorem allows us to study $\ell(G)$ in a slightly more general context. A graph is near-cubic if every vertex has degree 3, except for at most one vertex of degree 2. Schönberger's theorem implies that every 2-connected near-cubic graph $G$ has a 2-factor, and thus $\ell(G)$ is defined for this wider class.

Jackson and Yoshimoto [3] prove a theorem that implies that a 3-connected cubic graph $G$ has a 2-factor in which every component has length at least 5. In particular, for a 3connected cubic graph $G, \ell(G) \geq 5$.

A near-cubic graph is cyclically $k$-connected if the removal of fewer than $k$ edges does not create a graph with cycles in two different components, and we let $\mathcal{C}_{k}(n)$ denote the class of all near-cubic cyclically $k$-connected graphs on $n$ vertices. Observe that, in a cubic graph, a minimal size edge-cut is either a matching or the 3 edges incident with a vertex, so that, for $k \leq 3$, the three notions of being cyclically $k$-connected, being $k$-edge connected, and being $k$-connected are all equivalent. Subdividing an edge does not affect cyclic connectivity, so that this notion is a more useful indicator of the structure of nearcubic graphs. We define $L_{k}(n)$ as the least value of $\ell(G)$, taken over all graphs $G$ in $\mathcal{C}_{k}(n)$.

The only cubic graphs on at most 6 vertices are the 3-connected graphs $K_{4}, K_{3,3}$ and the triangular prism $K_{3} \square K_{2}$. All of these are Hamiltonian, as are all other 2-connected cubic graphs on 8 or 10 vertices, with the exception of the Petersen graph $\mathbb{P}$ (see Figure 2.) It is also not hard to see that the near-cubic graphs $G^{*}$ obtained by subdividing any 2connected cubic graph $G$ on at most 10 vertices other than $\mathbb{P}$ is also Hamiltonian, and it follows that $L_{2}(n)=n$ for $4 \leq n \leq 9$, whereas $L_{2}(10)=5$ and $L_{2}(11)=6$. With some more case analysis it can be shown that $L_{2}(n)=n-5$ for $10 \leq n \leq 16$, except $L_{2}(15)=9$, and the constructions achieving these values are given at the end of the proof of Theorem 2.1. For general $k$, observe that any $G \in \mathcal{C}_{k}(n)$ (with the possible exception of $K_{4}$ and $K_{3,3}$ ) has girth at least $k$, and thus $\ell(G) \geq k$ and $n \geq 2 k$. By considering an arbitrary 2-factor, it is not hard to see that $L_{k}(n)=n$ for $n=2 k$, except for $\mathbb{P}$ yielding $L_{5}(10)=5$.

The obvious question is, what is the behavior of $L_{2}(n)$, and more generally $L_{k}(n)$, as $n$ tends to infinity. In Section 2, we show that, for all $n, L_{2}(n) \leq 11$ and $L_{3}(n) \leq 16$. In Section 3 we prove that, for all $n \geq 12, L_{2}(n) \geq 7$.

Our consideration of the parameter $L_{3}(n)$ arose from the question: how does Petersen's Theorem generalize to infinite graphs? Tutte [8] proved his 1-factor theorem for locally finite graphs, so the modern proof of Petersen's Theorem from Tutte's Theorem applies to show that every 2-connected cubic graph has a 2-factor. In the infinite case, we were initially interested in whether there is a 2-factor in which every component is finite or whether there is a 2-factor in which every component is infinite. Thomassen (personal communication) gave us an example of a 2-connected cubic graph with infinitely many ends so that every 2 -factor must have an infinite component. The construction showing $L_{3}(n) \leq 16$ provides an infinite (1-ended) 3 -connected cubic graph in which every 2 -factor has only cycles of length at most 16 . In Section 4 , we provide an example of an infinite (1-ended) 3-connected cubic graph in which every 2-factor is a 2-way infinite Hamilton 
path.

\section{Upper bounds}

In this section we prove that $L_{2}(n) \leq 11$ and $L_{3}(n) \leq 16$.

For our constructions we first need some simple observations about the Petersen graph $\mathbb{P}$ : every 2-factor in $\mathbb{P}$ consists of two 5-cycles, and every 5-cycle is in a unique 2-factor. The edges of $\mathbb{P}$ partition into five sets of three edges, no two of which are together in any path of length 3 in $\mathbb{P}$; any 2-factor uses precisely two edges from each of the five sets and the two edges from the same set are in distinct components of the 2-factor.

The 2-merge of a graph $G$ (at an edge $x y$ of $G$ ) with a graph $H$ (at an edge $u v$ of $H$ ) means that we take disjoint copies of $G$ and $H$ and replace the edges $x y$ and $u v$ with the edges $x u$ and $y v$. If $G$ and $H$ are 2-connected near-cubic graphs, then their 2-merge is also 2-connected and near-cubic, as long as at least one of $G$ and $H$ is cubic.

If $G$ is a cubic graph, then the graph $G^{*}$ is obtained from $G$ by subdividing one edge of $G$. If $G$ is a graph and $w$ is a vertex of degree 2 in $G$, then suppressing $w$ results in the graph $G \diamond w$ obtained from $G-w$ by adding in an edge joining the two neighbours of $w$. If $W$ is a set $\left\{w_{1}, w_{2}, \ldots, w_{k}\right\}$ of vertices, all with degree 2 in $G$, then suppressing $W$ results in the graph $\left.\left(\cdots\left(G \diamond w_{1}\right) \diamond w_{2}\right) \diamond \cdots\right) \diamond w_{k}$. We will be careful to only suppress vertices in situations when no parallel edges can arise.

For "large" values of $n$ we have the following upper bounds; we conjecture equality holds for $n$ sufficiently large.

Theorem 2.1. $L_{2}(n) \leq 11$ when $n$ is congruent to 2 or 6 modulo 10 , and $L_{2}(n) \leq 10$ otherwise.

Proof. The result is obviously true for $n \leq 10$, and follows for $n=11$ by the subdivided Petersen graph $\mathbb{P}^{*}$, and for $n=12$ by replacing a vertex of $\mathbb{P}$ by a triangle. Thus, we may assume $n>12$.

Consider $n=10 k$ for some $k \geq 1$. Take disjoint copies $G_{1}, \ldots G_{k}$ of $\mathbb{P}$, each with two specified edges $u_{i} v_{i}$ and $x_{i} y_{i}$ of $G_{i}$ not in the same cycle of any 2-factor of $G_{i}$. We iteratively 2-merge all the $G_{i}$, starting with the 2-merge $G_{1}$ (at $x_{1} y_{1}$ ) with $G_{2}\left(\right.$ at $\left.u_{2} v_{2}\right)$, then 2-merging this (at $x_{2} y_{2}$ ) with $G_{3}\left(\right.$ at $u_{3} v_{3}$ ), and in general merging $G_{i}$ at $x_{i} y_{i}$ with $G_{i+1}$ at $u_{i+1} v_{i+1}$. The resulting graph $H(k)$ is a cubic 2-connected graph on $n$ vertices.

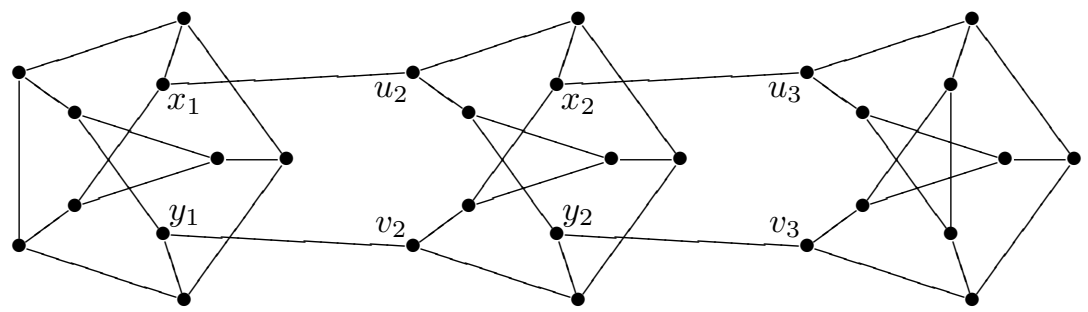

Figure 1: $H(3)$.

Claim 1. For $k \geq 2$, $\ell(H(k))=10$.

Proof. It is easy to see that any 2-factor of $H(k)$ arises from a 2-factor of the $G_{i}$ 's by doing the specified edge replacements where necessary, and thus the length of each cycle 
in a 2-factor of $H(k)$ is a multiple of 5. Taking a 2-factor in each $G_{i}$ containing both of the specified edges, we obtain a 2 -factor of $H(k)$ consisting of two 5-cycles (one each in $G_{1}$ and $\left.G_{k}\right)$ and $k-110$-cycles, so that $\ell(H(k)) \geq 10$.

To see that equality holds, observe that any 2-factor $F$ of $H(k)$ induces a 2-factor $F_{i}$ in each $G_{i}$. Each $F_{i}$ has two cycles of length 5 and none uses both edges that are switched to connect $G_{i}$ with $G_{i-1}$ and $G_{i+1}$. Thus, each component of $F$ can intersect at most two consecutive $G_{i}$ and, therefore, has length at most 10 .

To complete the proof of Theorem 2.1, let $n=10 k+r$, with $1 \leq r \leq 9$.

Case $r=6$. In this case 2-merge $H(k)$ at $u_{1} v_{1}$ with $K_{3,3}$, which at the most can lengthen the 5-cycle in $H(k)$ through $u_{1} v_{1}$ by six vertices to an 11-cycle, and hence $L(10 k+6) \leq$ 11.

Case $r=2$. Here we 2-merge $H(k-1)$ with a $K_{3,3}$ each at $u_{1} v_{1}$ and $x_{k-1} y_{k-1}$ to obtain $L(10(k-1)+12) \leq 11$, since here $k \geq 2$.

The following construction is useful in many of the remaining cases. The $(2 k-1)$ replacement in $G$ at the edge $w_{1} z_{1}$, is obtained by subdividing the edge $w_{1} z_{1} 2 k-1$ times, thereby creating the path $\left(w_{1}, w_{2}, \ldots, w_{k}, v, z_{k}, z_{k-1}, \ldots, z_{1}\right)$, and then adding all the edges $w_{i} z_{i}, i=2,3, \ldots, k$. It is easy to see that, if $k \geq 2$, for any $(2 k-1)$-replacement $G^{\prime}$ in $G$ and any 2-factor $F$ of $G$, there is a 2-factor $F^{\prime}$ that naturally extends $F$; in particular, $\ell\left(G^{\prime}\right) \geq \ell(G)$. In the case $k=1$, the $(2 k-1)$-replacement is simply subdividing $w_{1} z_{1}$.

If $r$ is not 2 or 6 , then there exist $s \in\{0,1,3,4,5\}$ and $t \in\{0,4\}$ such that $r=s+t$. Start with $H(k)$. If $t=4$, then 2-merge $H(k)$ at $x_{k} y_{k}$ with a $K_{4}$ to obtain a graph $G^{\prime}$ on $10 k+t$ vertices. If $k \geq 2$, then $\ell\left(G^{\prime}\right)=10$, while for $k=1 \ell\left(G^{\prime}\right)=9$. If $s \in\{1,3,5\}$, $s$-replace $u_{1} v_{1}$, while if $s=4$ we 2-merge $G^{\prime}$ at $u_{1} v_{1}$ with a $K_{4}$. None of these operations increases $\ell$ beyond 10 .

To obtain a construction for the 3-connected case, let $w, z$ be non-adjacent vertices in $\mathbb{P}$, let $c$ be their common neighbor, and let the remaining neighbors of $w$ be $u, v$ and the remaining neighbors of $z$ be $x, y$, where $u x$ and $v y$ are edges of $\mathbb{P}$. Observe that every 5-cycle in $\mathbb{P}$ that contains $w$ and $z$ also contains $c$ and exactly one of $u x$ and $v y$. Moreover, $\mathbb{P}-z$ has exactly two 2-factors: a 9-cycle through $w c$ and $w u$ and a 9-cycle through $w c$ and $w v$.

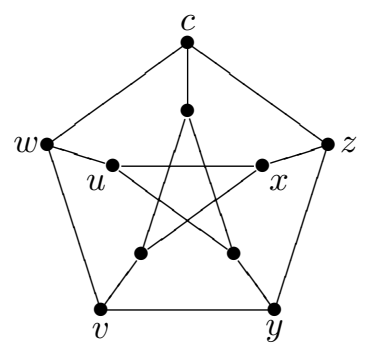

Figure 2: Petersen graph $\mathbb{P}$.

A 3-merge of a cubic graph $G$ (at a vertex $w$ in $G$ ) with a near-cubic graph $H$ (at a degree 3 vertex $z$ of $H$ ) is obtained from disjoint copies of $G-w$ and $H-z$ by adding a 
matching $u_{1} x_{1}, u_{2} x_{2}, u_{3} x_{3}$ from the neighborhood $\left\{u_{1}, u_{2}, u_{3}\right\}$ of $w$ to the neighborhood $\left\{x_{1}, x_{2}, x_{3}\right\}$ of $z$. The resulting near-cubic graph is cyclically 3 -connected if and only if both $G$ and $H$ are.

Theorem 2.2. $L_{3}(n) \leq 16$.

Proof. The result is obviously true for $n \leq 16$.

We start by considering the case $n=8 k+2, k \geq 2$. Take disjoint copies $G_{1}, \ldots G_{k}$ of $\mathbb{P}$, each with the specified vertices $u_{i}, v_{i}, w_{i}, c_{i}, x_{i}, y_{i}, z_{i}$ as above. We obtain the graph $J(k)$ by iteratively 3-merging the $G_{i}$, beginning with $G_{1}$ (at $z_{1}$ ) and $G_{2}$ (at $w_{2}$ ), and then 3-merging this (at $z_{2}$ ) with $G_{3}$ (at $w_{3}$ ), and so on. The specific 3-merges we use include the edges in $M_{i}=\left\{c_{i} u_{i+1}, x_{i} v_{i+1}, y_{i} c_{i+1}\right\}$ (see Figure 3.)

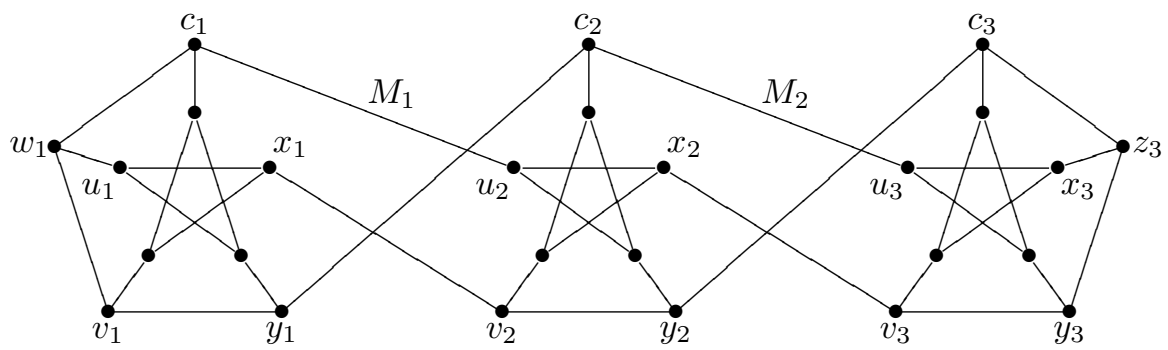

Figure 3: $J(3)$.

Claim 1. Suppose $k \geq 3$ and $F$ is a 2-factor of $J(k)$. If, for some $i$ with $1<i<k, C$ is a cycle in $F$ meeting both $M_{i-1}$ and $M_{i}$, then $y_{i-1} c_{i}$ and $c_{i} u_{i+1}$ are both edges of $C$, and $V(C) \cap V\left(G_{i}\right)$ is $\left\{c_{i}, u_{i}, x_{i}\right\}$ or $\left\{c_{i}, v_{i}, y_{i}\right\}$.

Proof. Because $F$ is a 2-factor, two edges incident with $c_{i}$ are in $F$. Thus, either $y_{i-1} c_{i}$ or $c_{i} u_{i+1}$ (or both) is in $F$. Since $C$ is the only cycle in $F$ that meets $M_{i-1} \cup M_{i}$ it follows that $c_{i}$ is in $C$. Obtain a 2-factor $F_{i}$ in $G_{i}$ from $F$ by deleting all of $G_{1}, \ldots, G_{i-1}$, $G_{i+1}, \ldots, G_{k}$, and adding back $w_{i}$ and $z_{i}$, together with the edges corresponding to those in $F \cap M_{i-1}$ as incident with $w_{i}$, and likewise with $z_{i}$. The cycle $C$ now corresponds to the 5-cycle $C_{i}$ in $F_{i}$ containing $c_{i}$.

As $C_{i}$ contains edges incident with both $w_{i}$ and $z_{i}$, the remark preceding Theorem 2.2 shows that $C_{i}$ contains exactly one of $u_{i} x_{i}$ and $v_{i} y_{i}$. Also, both $w_{i} c_{i}$ and $c_{i} z_{i}$ are in $C_{i}$ and, therefore, $y_{i-1} c_{i}$ and $c_{i} u_{i+1}$ are in $C$ and $V(C) \cap V\left(G_{i}\right)$ is either $\left\{c_{i}, u_{i}, x_{i}\right\}$ or $\left\{c_{i}, v_{i}, y_{i}\right\}$, as required.

Claim 2. If $C$ is a cycle of a 2-factor $F$ of $J(k)$ that is contained in at most two $G_{i}$, then $C$ has length at most 16.

Proof. If $C$ is contained in a single $G_{i}$, then obviously $C$ has length at most 9 (which could occur for $G_{1}$ or $G_{k}$ ). If $C$ meets both $G_{i}$ and $G_{i+1}$, then $C$ has length at most 16: this is obvious if $1<i<k-1$, while in the remaining case, $C$ can have at most 4 vertices in either $G_{1}$ or $G_{k}$ and, therefore, has length at most 12 .

Claim 3. For $k \geq 4, \ell(J(k))=16$. 
Proof. It is easy to see that $\ell(J(k)) \geq 16$ : pick the 9-cycle in $G_{1}-z_{1}$, a cycle of length 16 using only vertices from $G_{2}$ and $G_{3}$, and any 2 -factor to cover the remainder. (If $k$ is even, then this can be done with two 9-cycles and $(k-2) / 216$-cycles; if $k$ is odd, then this can be done with one 9-cycle, $(k-3) / 2$ 16-cycles, one 12-cycle, and one 5-cycle.)

For the reverse inequality, let $C$ be a cycle of a 2-factor $F$ of $J(k)$. By Claim 2, if $C$ is contained in at most two consecutive $G_{i}$, then $C$ has length at most 16 . Thus, we may assume that $C$ meets all of $G_{i-1}, G_{i}$, and $G_{i+1}$. From Claim 1, we see that $c_{i}, y_{i-1}$, and $u_{i+1}$ are in $C$, and that $C$ meets $G_{i}$ in exactly 3 vertices.

Suppose first that neither $c_{i-1}$ nor $c_{i+1}$ is in $C$. Then Claim 1 implies $C$ meets neither $M_{i-2}$ or $M_{i+2}$. In this case (or if $i=2$ or $i=k-1$ ), $C$ meets both $G_{i-1}$ and $G_{i+1}$ in precisely four vertices, as the cycles containing $c_{i-1}$ and $c_{i+1}$ are different from $C$. As there are three vertices of $G_{i}$ in $C, C$ has exactly 11 vertices.

Suppose $C$ contains $c_{i-1}$. We claim $c_{i+1}$ is not in $C$. To see this, observe that Claim 2 implies $c_{i-1} u_{i}$ and $y_{i-1} c_{i}$ are in $C$, as is $u_{i} v_{i}$. This implies that $y_{i}$ is not in $C$, so Claim 2 implies $c_{i+1}$ is not in $C$.

It follows that $C$ meets $G_{i+1}$ in four vertices. Likewise, $C$ meets $G_{i-2}$ (assuming $i \geq 3$ ) in four vertices, for a total length of 14 .

Suppose $n=8 k+2+r$, for $k \geq 2$ and $0 \leq r \leq 7$. We exhibit a graph $J_{r}(k)$ so that $\ell\left(J_{r}(k)\right) \leq 16$. We start with $J_{0}(k)=J(k)$. Observe that the same argument as above shows that if a cycle in a 2 -factor of $J(k)$ contains exactly one of $w_{1}$ or $z_{k}$, then its length is at most 12, whereas if it contains both, then it has length at most 8 (and $k \leq 3$ ).

We get $J_{1}(k)$ by subdividing an edge of $J(k)$ incident with $w_{1}$. When $2 \leq r \leq 4$, $J_{r}(k)$ is a 3-merge of $J(k)$ at $w_{1}$ with any graph in $\mathcal{C}_{3}(r+2)$.

When $5 \leq r \leq 7, J_{r}(k)$ is the 3-merge of $J_{r-4}(k)$ at $z_{k}$ with $K_{3,3}$. The only remaining case is $n=17$, in which case we take a 3 -merge of $\mathbb{P}$ with any graph in $\mathcal{C}_{3}(9)$ to see that $L_{3}(17) \leq 12$.

\section{Lower bound}

The previous section shows that $L_{2}(n) \leq 11$. In this section, we show that $L_{2}(n) \geq 7$ for $n \geq 12$. More precisely, we prove the following.

Theorem 3.1. If $G$ is a 2-connected near-cubic graph, then either $\ell(G) \geq 7$ or $\ell(G)=$ $|V(G)|$ or $G$ is $\mathbb{P}$ or $G$ is $\mathbb{P}^{*}$.

The proof will be by induction. We start with some useful terminology and basic lemmas that will serve as reductions in the proof of Theorem 3.1.

We always let $t$ be the vertex of degree 2 in $G$, if it exists. Given an edge $e$ in a nearcubic graph $G$, let $\ell(G, e)$ denote the length of a longest cycle through $e$ that is contained in a 2 -factor of $G$. The first lemma yields the base case for our induction, and will help in many of the reductions. It can be verified by checking all small cases.

Lemma 3.2. Let $n \leq 10$ and let $G$ be a 2-connected near-cubic graph on $n$ vertices.

1. $\ell(G)=n$, except if $G=\mathbb{P}$.

2. If $G$ is cyclically 3-connected, then, for each edge e of $G, \ell(G, e)=\ell(G)$. 
Observe that the smallest near-cubic graph with cyclic connectivity 2 is the Hamiltonian graph $G$ on 7 vertices obtained from a 3-replacement of $K_{4}$, but that the edge $w_{2} z_{2}$ is in no Hamilton cycle of $G$. Recall (see the paragraph following the case $r=2$ in the proof of Theorem 2.1) that any $(2 k-1)$-replacement of an edge in a cubic graph still has a 2 -factor.

Lemma 3.3. If $G$ is the $(2 k-1)$-replacement of the edge e in a 2-connected cubic graph $G^{\prime}$, then $\ell(G) \geq \ell\left(G^{\prime}, e\right)+(2 k-1)$ when $k \geq 1$. If $k \geq 2$, then also $\ell(G) \geq \ell\left(G^{\prime}\right)$.

Proof. A 2-factor in $G^{\prime}$ that contains a cycle $C$ through $e$ can be extended to a 2-factor of $G$ by including the new vertices in $C$, immediately yielding the first bound. If a longest 2 -factor of $G^{\prime}$ does not use the edge $e$, then, when $k \geq 2$, it can still be extended to a 2 -factor of $G$ by including a new cycle consisting of all the replacement vertices.

Given disjoint induced subgraphs $H, K$ of a graph $G$ so that $V(G)=V(H) \cup V(K)$, we use $[H, K]$ to denote the set of edges having one end in each of $H$ and $K$, that is, the (edge) cut that separates $H$ from $K$. Moreover, $[H, K]$ is a $k$-cut, provided that its size $|[H, K]|$ is $k$.

A $k$-cut $[H, K]$ in a 2-connected near-cubic graph is cyclic if both $H$ and $K$ contain a cycle; otherwise it is non-cyclic. It is easy to see that if $[H, K]$ is a non-cyclic 2-or 3-cut, with $H$ not containing a cycle, then $H$ is either a single vertex of degree $|[H, K]|$ or $H$ consists of two adjacent vertices, one of which is the degree 2 vertex $t$ of $G$. In general the edges in a cyclic cut of minimum size in a near-cubic graph $G$ form a matching.

In order to deal with 2-cuts, we need to understand their structure in more detail. Let $\left(u_{0}, u_{1}, \ldots, u_{k}\right)$ and $\left(v_{0}, v_{1}, \ldots, v_{k}\right)$ be disjoint paths in $G$ so that, for each $i$ with $0<$ $i<k, u_{i}$ and $v_{i}$ are adjacent in $G$. Then the union of the two paths and the edges $u_{i} v_{i}$, $i=1,2, \ldots, k-1$ is a $\left(u_{0}, u_{k}, v_{0}, v_{k}\right)$-ladder in $G$. The two paths are the rails of the ladder and the edges $u_{i} v_{i}$ are its rungs.

Let $\left[H^{\prime}, K^{\prime}\right]$ be a 2-cut in $G$ such that $H^{\prime}$ contains the degree 2 vertex $t$, if it exists. If $t$ is in a triangle, then our earlier remarks (see the paragraph following the case $r=2$ in the proof of Theorem 2.1) show $G$ is a $(2 k-1)$-replacement in some cubic graph, and $k \geq 2$. If $G$ is cubic, then [9, Lemma 7.3.3.] shows there are induced subgraphs $H$ and $K$ of $H^{\prime}$ and $K^{\prime}$, respectively, with distinct vertices $u_{H}, v_{H}$ of $H^{\prime}$, not adjacent in $H$, and $u_{K}, v_{K}$ of $K^{\prime}$, not adjacent in $K$, and there is a $\left(u_{H}, u_{K}, v_{H}, v_{K}\right)$-ladder $L$ in $G$ so that $G=H \cup L \cup K$. Moreover $H$ and $K$ have at least four vertices each. In the remaining case that $t$ exists and is not in a triangle, the preceding sentence applies to the graph obtained by suppressing $t$, and we may restore $t$ in $H$, by possibly shortening the ladder if $t$ is in $L$. The decomposition $G=H \cup L \cup K$ of $G$ is a cyclic 2-cut ladder decomposition.

The graphs $H_{(2)}$ and $K_{(2)}$ are obtained from $H$ and $K$, respectively by adding the edges $e_{H}=u_{H} v_{H}$ and $e_{K}=u_{K} v_{K}$, respectively. Observe that $H_{(2)}$ and $K_{(2)}$ are near-cubic and 2-connected.

Lemma 3.4. Suppose $G$ is a 2-connected near-cubic graph that has a cyclic 2-cut ladder decomposition $H \cup L \cup K$. Specifically, the vertex $t$, if it exists, is not in a triangle, but is in $H$. Let the rails of $L$ have length $k$.

1. $\ell(G) \geq \ell\left(H_{(2)}, e_{H}\right)+\ell\left(K_{(2)}, e_{K}\right)+2 k-2$.

2. $\ell(G) \geq \ell\left(H_{(2)}\right)$. 
Proof. Suppose that $F$ is a 2-factor of $H_{(2)}$ containing $e_{H}$ in the cycle $C$ and that $F^{\prime}$ is a 2-factor of $K_{(2)}$ containing $e_{K}$ in the cycle $C^{\prime}$. Obtain a 2-factor of $G$ from $\left(F \backslash\left\{e_{H}\right\}\right) \cup$ $\left(F^{\prime} \backslash\left\{e_{K}\right\}\right)$ by adding the rails of $L$. The cycle of this 2-factor of $G$ that contains the ladder rails has length $|V(C)|+\left|V\left(C^{\prime}\right)\right|+2 k-2$.

For the second part, let $F$ be a longest 2-factor of $H_{(2)}$. If some cycle $C$ of $F$ contains $e_{H}$, then consider any 2-factor of $K_{(2)}$ that contains $e_{K}$ and find a 2-factor for $G$ as above. Observe that the longest cycle in $H_{(2)}$ either remains unchanged or is extended, so we are done.

Finally, suppose that $e_{H}$ is not in $F$. If $k=1$, then let $F^{\prime}$ be a 2-factor of $K_{(2)}$ not using $e_{K}$, and now $F \cup F^{\prime}$ is a 2-factor of $G$. If $k>1$, then let $F^{\prime}$ be a 2-factor of $K_{(2)}$ using $e_{K}$ in some cycle $C$, and convert $F^{\prime}$ into a 2-factor $F^{\prime \prime}$ of $G-V(H)$ by including the ladder vertices in $C$. Now $F \cup F^{\prime \prime}$ is a 2-factor of $G$. In either case, the 2-factor of $G$ contains $F$ and the second part follows.

The next lemma helps us to deal with cyclic 3-cuts $[H, K]$.

Lemma 3.5. Suppose $G$ is a 3-merge of a 2-connected near-cubic graph $H_{(3)}$ at $v_{H}$ and a 2-connected cubic graph $K_{(3)}$ at $v_{K}$.

1. Then $\ell(G) \geq \ell\left(H_{(3)}\right)$.

2. If there is a 2-factor $F$ of $H_{(3)}$ so that the cycle of $F$ through $v_{H}$ has length $\ell\left(H_{(3)}\right)$, then $\ell(G) \geq \ell\left(H_{(3)}\right)+1$.

Proof. Let $F$ be a longest 2-factor of $H_{(3)}$ and suppose $F$ does not use the edge $e$ incident with $v_{H}$. There is a 2-factor $F^{\prime}$ of $K_{(3)}$ that does not contain the edge incident with $v_{K}$ that corresponds to $e$. $F$ and $F^{\prime}$ combine to produce a 2-factor of $G$, where the cycle $C$ of $F$ through $v_{H}$ has merged with the cycle $C^{\prime}$ of $F^{\prime}$ through $v_{K}$ to produce a cycle of length $|V(C)|-1+\left|V\left(C^{\prime}\right)\right|-1 \geq|V(C)|+1$; all other cycles of $F$ remain unaffected, and the results follow.

Lemma 3.6. Let $G$ be a cyclically 4-connected near-cubic graph with $|V(G)| \geq 10$ and a 4-cycle $C=\left(v_{0}, v_{1}, v_{2}, v_{3}, v_{0}\right)$. (Throughout, all indices are modulo 4.) Then:

1. G has no triangle;

2. each vertex $v_{i}$ in $C$ has a distinct neighbor $w_{i}$ not in $C$;

3. for each $i \in\{1,2\}, G-\left\{v_{i} v_{i+1}, v_{i+2} v_{i+3}\right\}$ is 2-connected;

4. for some $i \in\{1,2\}$, suppressing the four degree 2 vertices $v_{1}, v_{2}, v_{3}$, and $v_{0}$ in $G-\left\{v_{i} v_{i+1}, v_{i+2} v_{i+3}\right\}$ produces a 2 -connected near-cubic graph $G^{\prime}$ with new edges $E\left(G^{\prime}\right)-E(G)=\left\{w_{i+1} w_{i+2}, w_{i+3} w_{i}\right\}$.

5. $\ell(G) \geq \ell\left(G^{\prime}\right)$ and if $e$ is a new edge, then $\ell(G) \geq \ell\left(G^{\prime}, e\right)+2$.

Proof. (1) Let $H$ be the subgraph of $G$ induced by the vertices in a triangle and let $K=$ $G-V(H)$. Then $[H, K]$ is a cyclic $k$-cut for some $k \in\{2,3\}$, a contradiction.

(2) $[C, G-V(C)]$ is a cyclic $k$-cut for some $k \leq 4$. Since $G$ is cyclically 4-connected, $k=4$ and the edges in the cut form a matching. Thus each $v_{i}$ has a distinct neighbor $w_{i}$ not in $C$.

(3) Let $e_{j}=v_{j} v_{j+1}$. Since $e_{i}$ and $e_{i+2}$ are not both incident with $t, G-\left\{e_{i}, e_{i+2}\right\}$ is connected. If it is not 2 -connected, then it must have a cut-edge $f$, and thus $\left\{e_{i}, e_{i+2}, f\right\}$ is 
a 3-cut in $G$. Since $G$ is cyclically 4 -connected, this cut must be non-cyclic, contradicting the fact that the vertices incident with $e_{i}$ and $e_{i+2}$ are all distinct and have degree 3 .

(4) Suppose the graph $G_{i}$ obtained from $G-\left\{e_{i}, e_{i+2}\right\}$ by suppressing the vertices in $C$ has a multiple edge. Then either $w_{i} w_{i+3}$ or $w_{i+1} w_{i+2}$ is an edge of $G$ and, after suppression, it is parallel to one of the paths $\left(w_{i}, v_{i}, v_{i+3}, w_{i+3}\right)$ and $\left(w_{i+1}, v_{i+1}, v_{i+2}, w_{i+2}\right)$. If this happens for both $G_{1}$ and $G_{2}$, then, for some $j \in\{0,1,2,3\}, w_{j}$ has the three neighbours $v_{j}, w_{j+1}$ and $w_{j-1}$. In this case, let $H$ be the subgraph of $G$ induced by $V(C) \cup\left\{w_{j}, w_{j+1}, w_{j-1}\right\}$ and let $K$ be the subgraph of $G$ induced by the remaining vertices of $G$. The choice of $H$ implies that $[H, K]$ is a $k$-cut for some $k \leq 3$, but since $|V(K)| \geq 10-7=3$ this cut is cyclic, a contradiction. Thus, we may assume that $G_{1}$ is a near-cubic graph. Suppressing degree 2 vertices does not decrease connectivity, so $G_{1}$ is 2-connected.

(5) We assume that $G_{1}$ is 2 -connected and simple and let $F$ be a 2 -factor of $G_{1}$. We reconstruct a 2 -factor of $G$ in each of the three resulting cases.

If $F$ uses neither of the new edges $w_{0} w_{1}$ and $w_{2} w_{3}$, then $F \cup\{C\}$ is a 2-factor in $G$.

If $F$ uses precisely one new edge, say $w_{0} w_{1}$, then let $C^{\prime}$ be the cycle in $F$ through this edge. In this case, we get a 2 -factor of $G$ by replacing $w_{0} w_{1}$ in $C^{\prime}$ with $\left(w_{0}, v_{0}, v_{3}, v_{2}\right.$, $\left.v_{1}, w_{1}\right)$.

Finally, if $F$ uses both $w_{0} w_{1}$ and $w_{2} w_{3}$, then we simply replace them with $\left(w_{0}, v_{0}\right.$, $\left.v_{1}, w_{1}\right)$ and $\left(w_{2}, v_{2}, v_{3}, w_{3}\right)$, respectively.

Lemma 3.7. Let $G$ be a cyclically 4-connected cubic graph.

1. Suppose $G$ has a 5-cycle $\left(v_{0}, v_{1}, v_{2}, v_{3}, v_{4}, v_{0}\right)$, and indices throughout are modulo 5. Then, for each $i$ with $0 \leq i \leq 4,\left(G-v_{i}\right)-v_{i+2} v_{i+3}$ is 2-connected. If $G$ has no 4 -cycles, then suppressing the four degree 2 vertices $v_{i+1}, v_{i+2}, v_{i+3}$ and $v_{i+4}$ in $\left(G-v_{i}\right)-v_{i+2} v_{i+3}$ produces a 2 -connected near-cubic graph.

2. Suppose $G$ has a 6 -cycle $\left(v_{0}, v_{1}, v_{2}, v_{3}, v_{4}, v_{5}, v_{0}\right)$, and indices throughout are modulo 6. Then, for each $i \in\{0,1\}$, the graph $G-\left\{v_{i} v_{i+1}, v_{i+2} v_{i+3}, v_{i+4} v_{i+5}\right\}$ is 2-connected. If $G$ has no 4-cycles, then suppressing the six degree 2 vertices in $G-\left\{v_{i} v_{i+1}, v_{i+2} v_{i+3}, v_{i+4} v_{i+5}\right\}$ produces a 2 -connected cubic graph.

Proof. (1) Let $C$ be the cycle $\left(v_{0}, v_{1}, v_{2}, v_{3}, v_{4}, v_{0}\right)$ and let $e_{i}$ be the edge $v_{i} v_{i+1}$. Since $G$ is cubic, and triangle-free by Lemma 3.6.1, each $v_{i}$ has a unique neighbor $w_{i}$ not in $C$.

As $G$ is 3-connected, $G-v_{i}$ is 2-connected, so $G-\left\{v_{i}, e_{i+2}\right\}$ is connected. If it is not 2-connected, then it has a cut-edge $f$. Let $H$ and $K$ be the two components of $G-\left\{v_{i}, e_{i+2}, f\right\}$. Choose the labelling of $H$ and $K$ so that $v_{i}$ has at most one neighbour $z$ in $H$. Consider the cut $\left[H, K+v_{i}\right]$ in $G$. Then $\left[H, K+v_{i}\right] \subseteq\left\{e_{i+2}, v_{i} z, f\right\}$; since $G$ is cubic and cyclically 4-connected, equality holds and all 3 edges must be incident with the same vertex. Since $v_{i}$ is not incident with $e_{i+2}$ this vertex can only be $z$, so that $v_{i} z$ is a chord of $C$, contradicting the fact that $G$ is triangle-free.

Finally, if $G$ has no 4-cycles, for each $i=0,1,2,3,4$, the $w_{i}$ are distinct and there is no edge of $G$ joining $w_{i}$ and $w_{i+1}$, which shows suppressing the four degree 2 vertices in $G-\left\{v_{i}, e_{i+2}\right\}$ results in a near-cubic 2 -connected graph, with degree 2 vertex $w_{i}$.

(2) Let $C$ be the cycle $\left(v_{0}, v_{1}, v_{2}, v_{3}, v_{4}, v_{5}, v_{0}\right)$ and let $e_{i}$ be the edge $v_{i} v_{i+1}$.

As $G$ is 3-connected, if $G-\left\{e_{i}, e_{i+2}, e_{i+4}\right\}$ is not connected, then there is a 3-cut $[H, K]$ so that $[H, K]=\left\{e_{i}, e_{i+2}, e_{i+4}\right\}$. As $G$ has no cyclic 3 -cuts, one of $H$ and $K$ is a 
single vertex. However, the edges $e_{i}, e_{i+2}$, and $e_{i+4}$ have no incident vertex in common, a contradiction. Thus, $G-\left\{e_{i}, e_{i+2}, e_{i+4}\right\}$ is connected.

If $G-\left\{e_{i}, e_{i+2}, e_{i+4}\right\}$ has a cut-edge $e$, then $G-\left\{e, e_{i}, e_{i+2}, e_{i+4}\right\}$ has precisely two (cyclic) components $H$ and $K$, and $[H, K]=\left\{e, e_{i}, e_{i+2}, e_{i+4}\right\}$. Since $G$ is cyclically 4-connected, the edges in this 4-cut form a matching. It follows that the edges $e_{i+1}, e_{i+3}$ and $e_{i+5}$ are in $G-[H, K]$ and so at least two of them are in the same one of $H$ and $K$, say $e_{i+1}$ and $e_{i+3}$ are in $H$. But this is impossible, as $e_{i+1}$ and $e_{i+3}$ are incident with different ends of $e_{i+2}$ and one of these is in $K$. Thus, $G-\left\{e_{i}, e_{i+2}, e_{i+4}\right\}$ is 2-connected.

If $G$ has no 3- or 4-cycles, then $C$ is an induced cycle and we let $w_{j}$ be the neighbour of $v_{j}$ not in $C$. Then $w_{j}$ and $w_{j+1}$ are distinct and not adjacent in $G$. This is enough to conclude that the paths $\left(w_{j}, v_{j}, v_{j+1}, w_{j+1}\right)$ do not become multiple edges after suppressing the vertices in $C$ from $G-\left\{e_{i}, e_{i+2}, e_{i+4}\right\}$, and hence the resulting graph is 2-connected and cubic.

Let $C$ be a cycle in a graph $G$. A partial 2-factor of $G$ with respect to $C$ is a 2-regular subgraph $F$ of $G$ such that $V(G)-V(F) \subseteq V(C)$, and $E(F) \cap E(C)$ is a matching.

Lemma 3.8. Suppose a near-cubic 2-connected graph $G$ has a partial 2-factor $F$ with respect to a cycle $C$. Let $|E(C) \cap E(F)|=k$ and let $s$ be the number of vertices in the subgraph induced by $C$ and the cycles in $F$ that meet $C$. If some longest cycle in $F$ misses $V(C)$ (for example when $k=0$ ) or $|V(C)|=2 k$, then $\ell(G) \geq \ell(F)$. If $k>0$, then $\ell(G) \geq s / k$.

Proof. If $|V(C)|=2 k$, then $F$ is a 2-factor of $G$, and the result is obvious. Otherwise $|V(C)|>2 k$ and we consider $F^{\prime}$ such that $E\left(F^{\prime}\right)$ is the symmetric difference of $E(F)$ and $E(C)$ : in $F^{\prime}$ each vertex in $V(F)-V(C)$ is incident with 2 edges from $F$ and each vertex in $V(C)-V(F)$ is incident with 2 edges from $C$; each vertex in $V(C) \cap V(F)$ must be incident with exactly one edge from $E(C) \cap E(F)$ (since these form a matching and $G$ is near-cubic). It follows that $F^{\prime}$ is a 2-factor of $G$. When $k=0, F^{\prime}=F \cup\{C\}$ and thus $\ell(G) \geq \ell\left(F^{\prime}\right) \geq \ell(F)$.

When $k>0$, let $C^{1}, C^{2}, \ldots, C^{r}$ be the cycles of $F$ that meet $C$ and let $G^{\prime}=C \cup C^{1} \cup$ $C^{2} \cup \cdots \cup C^{r}$. Then $G^{\prime}$ is a 2 -connected sub-cubic graph on $s$ vertices with exactly $2 k$ vertices of degree 3. Observe that $F^{\prime \prime}=F^{\prime} \cap V\left(G^{\prime}\right)=G^{\prime}-(E(C) \cap E(F))$ is a 2-factor of $G^{\prime}$. Since $G^{\prime}$ is 2-connected each component of $F^{\prime \prime}$ has at least 2 vertices of degree 3 in $G^{\prime}$, and thus $F^{\prime \prime}$ has at most $k$ components. Thus one of the cycles in $F^{\prime \prime}$ has length at least $s / k$, and hence $\ell(G) \geq \ell\left(F^{\prime}\right) \geq \ell\left(F^{\prime \prime}\right) \geq s / k$.

We are now ready for the proof of the main result of this section. A 2-factor $F$ in a graph $G$ has a long cycle if one of the components of $F$ has length at least 7.

Proof of Theorem 3.1. We proceed by induction, with the base cases $|V(G)| \leq 10$ covered by Lemma 3.2. For the induction step, we may suppose $|V(G)| \geq 11$. Let $t$ be the degree 2 vertex, if $G$ has one.

Claim 1. If $t$ is in a triangle of $G$, then $\ell(G) \geq 7$.

Proof. There is a $k \geq 2$ so that $G$ is a $(2 k-1)$-replacement of some edge $e$ in a 2-connected cubic graph $G^{\prime}$. By Lemma 3.3, $\ell(G) \geq \ell\left(G^{\prime}\right)$, so the result follows unless $G^{\prime}$ either is $\mathbb{P}$ or has at most 6 vertices. In these cases, Lemma 3.2 implies $\ell\left(G^{\prime}, e\right)=\ell\left(G^{\prime}\right) \geq 4$, so Lemma 3.3 implies $\ell(G) \geq \ell\left(G^{\prime}, e\right)+(2 k-1) \geq 4+3=7$. 
Claim 2. If $G$ has a cyclic 2 -cut, then $\ell(G) \geq 7$.

Proof. From Claim 1, we may assume that either $G$ is cubic, or obtained by subdividing an edge of a cubic graph.

Since $G$ has a cyclic 2-cut, there are disjoint subgraphs $H$ and $K$ of $G$ and a ladder $L$ so that $G=H \cup L \cup K$, and $t$, if it exists, is in $H$. If $\ell\left(H_{(2)}\right) \geq 7$, then Lemma 3.4.2 shows that $\ell(G) \geq 7$, as required. Thus, we may assume $\ell\left(H_{(2)}\right)<7$.

We know that $H_{(2)}$ is either $\mathbb{P}$ or $\mathbb{P}^{*}$, or has at most 6 vertices. In any of these cases Lemma 3.2 implies that $\ell\left(H_{(2)}, e_{H}\right)=\ell\left(H_{(2)}\right) \geq 4$. Thus Lemma 3.4.1 implies $\ell(G) \geq$ $\ell\left(H_{(2)}, e_{H}\right)+\ell\left(K_{(2)}, e_{K}\right)+2 k-2 \geq 4+3+0=7$.

Claim 3. If $G$ is not cyclically 4-connected, then $\ell(G) \geq 7$.

Proof. From Claims 1 and 2, we may assume $G$ is cyclically 3-connected. Since $G$ is not cyclically 4-connected, $G$ has a cyclic 3 -cut $[H, K]$, with the labelling chosen so that $t$, if it exists, is in $H$. Since $[H, K]$ is a minimum size cyclic cut it must be a matching, and thus the graph $H_{(3)}$ obtained from $H$ by adding a new degree 3 vertex $v_{H}$ adjacent to the vertices in $H$ that are incident with edges in $[H, K]$ is simple near-cubic and 2-connected. Similarly the graph $K_{(3)}$ obtained from $K$ by introducing a new vertex $v_{K}$ is cubic 2-connected, and $G$ is the 3-merge of $H_{(3)}$ and $K_{(3)}$.

If $\ell\left(H_{(3)}\right) \geq 7$, then Lemma 3.5.1 implies that $\ell(G) \geq 7$, as required. Thus, we may assume $\ell\left(H_{(3)}\right)<7$. If $H_{(3)}$ is either $\mathbb{P}^{*}$, or has 6 vertices, then Lemma 3.5.2 implies (in the case of $\mathbb{P}^{*}$ because any vertex and any edge of $\mathbb{P}$ are in a 5-cycle in $\left.\mathbb{P}\right) \ell(G) \geq 6+1=7$. Thus, $H_{(3)}$ is $\mathbb{P}, K_{4}$ or $K_{4}^{*}$. If $H_{(3)}$ is cubic, then the roles of $H$ and $K$ can be reversed, so that $K_{(3)}$ must also be $K_{4}$ or $\mathbb{P}$. So, since $|V(G)| \geq 11$, it follows that $G$ is the 3 -merge of $\mathbb{P}$ and either $K_{4}$ or $\mathbb{P}$, whence $\ell(G) \geq 9$. Hence it remains to consider the case $H_{(3)}=K_{4}^{*}$.

In this case, let $u_{1}, u_{2}, u_{3}$ be the neighbors of $v_{H}$ in $H_{(3)}$, and let $v_{1}, v_{2}, v_{3}$ be their corresponding neighbors in $K$. Suppose the neighbors of $v_{H}$ in a Hamilton cycle $D$ in $H_{(3)}$ are $u_{i}, u_{j}$. Let $F_{k}$ be a 2-factor of $K_{(3)}$ avoiding $v_{K} v_{k}$, where $k \in\{1,2,3\} \backslash\{i, j\}$ and let $C_{k}$ be the cycle of $F_{k}$ through $v_{K}$. Then $F_{k}-C_{k}$ together with the cycle $C=$ $\left(D-v_{H}\right) \cup\left(C_{k}-v_{K}\right) \cup\left\{u_{i} v_{i}, u_{j} v_{j}\right\}$ is a 2-factor of $G$. As long as $C_{k}$ has length at least $4, C$ has length at least 7 . Thus, we may assume $C_{k}$ is the 3 -cycle $\left(v_{K}, v_{i}, v_{j}, v_{K}\right)$. In particular, $v_{i} v_{j}$ is an edge of $G$.

However $H_{(3)}$ has two different Hamilton cycles $D$, so we can assume that, say $v_{1} v_{2}$ and $v_{2} v_{3}$ are edges of $G$. There are at least three cubic vertices of $G$ not in $H \cup\left\{v_{1}, v_{2}, v_{3}\right\}$, so the 2-cut $\left[H \cup\left\{v_{1}, v_{2}, v_{3}\right\}, K-\left\{v_{1}, v_{2}, v_{3}\right\}\right]$ is cyclic, contradicting the assumption that $G$ is cyclically 3 -connected.

From now on we may assume that $G$ is cyclically 4-connected. In particular, $G$ is triangle-free by Lemma 3.6.1.

Claim 4. If $G$ has a 4-cycle, then $\ell(G) \geq 7$.

Proof. Let $C$ be a 4-cycle $\left(v_{0}, v_{1}, v_{2}, v_{3}, v_{0}\right)$ in $G$. Since $G$ is cyclically 4-connected, Lemma 3.6.2 implies that, for every $i \in\{0,1,2,3\}, v_{i}$ has a unique neighbor $w_{i}$ not in $C$. Note that, for $i=0,1,2,3, w_{i} \neq w_{i+1}$ (indices being read modulo 4). By Lemma 3.6.4, for some $i \in\{0,1\}$, the result of suppressing the four degree 2 vertices $v_{0}, v_{1}, v_{2}$, and $v_{3}$ in $G-\left\{v_{i} v_{i+1}, v_{i+2} v_{i+3}\right\}$ is a 2-connected graph $G^{\prime}$. 
Obviously $\left|V\left(G^{\prime}\right)\right|=|V(G)|-4 \geq 7$. If $\ell\left(G^{\prime}\right) \geq 7$, then the result follows from the first part of Lemma 3.6.5. Otherwise $G^{\prime}$ is $\mathbb{P}$ or $\mathbb{P}^{*}$, and Lemmas 3.2.2 and 3.6.5 imply $\ell(G) \geq \ell\left(G^{\prime}\right)+2 \geq 7$.

Perhaps surprisingly, we next treat two cases in which $G$ has a 6-cycle. Because there are no 3- or 4-cycles, any 6-cycle is induced.

Claim 5. If there is a 6-cycle $C$ in $G$ containing $t$, then $G=\mathbb{P}^{*}$ or $\ell(G) \geq 7$.

Proof. Let $C=\left(v_{0}, v_{1}, v_{2}, v_{3}, v_{4}, v_{5}, v_{0}\right)$, with the labelling chosen so that $v_{0}=t$. For $1 \leq i \leq 5$, let $w_{i}$ be the neighbour of $v_{i}$ not in $C$. Since $G$ has no 3-or 4-cycles, if $w_{i}=w_{j}$, then either $i=j$ or $i=j \pm 3$. Furthermore, we cannot have both $w_{1}=w_{4}$ and $w_{2}=w_{5}$, as then there is a cyclic 3-cut with $V(H)=V(C) \cup\left\{w_{1}, w_{2}\right\}$. So we may assume $w_{2} \neq w_{5}$. Also, for $i=1,2,3,4, w_{i}$ and $w_{i+1}$ are not adjacent in $G$.

Let $G^{\prime \prime}=G-\left\{v_{0}, v_{3}\right\}$. Suppressing $v_{0}$ in $G$ produces a graph to which Lemma 3.7.1 applies, showing $G^{\prime \prime}$ is 2-connected. Furthermore, suppressing the four degree 2 vertices $v_{1}, v_{2}, v_{4}, v_{5}$ in $G^{\prime \prime}$ yields a 2-connected near-cubic graph $G^{\prime}$ (since consecutive $w_{i}$ 's are not adjacent) with degree 2 vertex $w_{3}$.

We note that $\left|V\left(G^{\prime}\right)\right|=|V(G)|-6 \geq 5$. As $G^{\prime}$ is not cubic, by induction either $G^{\prime}=K_{4}^{*}$ or $G^{\prime}=\mathbb{P}^{*}$ or $\ell\left(G^{\prime}\right) \geq 7$.

Suppose first that $\left|V\left(G^{\prime}\right)\right|=5$. If $w_{1} \neq w_{4}$, then the five vertices of $G^{\prime}$ are $w_{1}, w_{2}$, $w_{3}, w_{4}$, and $w_{5}$. As $w_{3}$ is the degree 2 vertex and $w_{3}$ is not adjacent to either $w_{2}$ or $w_{4}$, the edges in $G-V(C)$ must be $w_{3} w_{5}, w_{3} w_{1}, w_{4} w_{2}, w_{4} w_{1}$ and $w_{2} w_{5}$, and thus $G=\mathbb{P}^{*}$. If $w_{1}=w_{4}$, then neither $w_{1}=w_{4}$ nor $w_{2}$ is adjacent to $w_{3}$ in $G^{\prime}$. Thus, the neighbors of $w_{3}$ are $w_{5}$ and some other vertex $x$. But then $w_{4}=w_{1}$ is adjacent to $w_{5}$ in $G$, a contradiction.

In the remaining case, either $G^{\prime}=\mathbb{P}^{*}$ or $\ell\left(G^{\prime}\right) \geq 7$. If the latter, then let $F^{\prime}$ be any longest 2-factor of $G^{\prime}$. If $G^{\prime}=\mathbb{P}^{*}$, then we claim there is a 2-factor $F^{\prime}$ of $G^{\prime}$ with a cycle $C^{\prime}$ through exactly one of $w_{4} w_{5}$ and $w_{1} w_{2}$. Indeed, if $w_{1}=w_{4}$, then observe that any $F^{\prime}$ containing the third edge incident with $w_{1}$ will avoid exactly one of these edges altogether. If $w_{1} \neq w_{4}$, then $w_{1} w_{2}$ and $w_{4} w_{5}$ are not incident with the same vertex of $G^{\prime}$ and so it is possible either to find a 6-cycle containing exactly one of these and $w_{3}$, or a 5-cycle containing only one of these together with a 6-cycle containing $w_{3}$.

Expanding $F^{\prime}$ back to $G$, we obtain a partial 2-factor $F$ of $G$ with respect to $C$. Set $k=|E(C) \cap E(F)|$ and let $s$ be the number of vertices in the subgraph induced by $C$ and the cycles in $F$ that meet $C$. Then $k=1$ when $G^{\prime}=\mathbb{P}^{*}$. By Lemma 3.8, $\ell(G) \geq \ell(F) \geq$ $\ell\left(F^{\prime}\right) \geq 7$, unless $1 \leq k \leq 2$ and a longest cycle $C^{\prime}$ of $F^{\prime}$ contains a new edge. Since $s \geq|V(C)|+\left|V\left(C^{\prime}\right)\right|$, it follows that, for $\ell\left(G^{\prime}\right) \geq 7$, we obtain $\ell(G) \geq(6+7) / k>6$, as desired. If $G^{\prime}=\mathbb{P}^{*}$, then $\ell(G) \geq(6+5) / 1=11$.

Claim 6. If $G$ is cubic and has a 6-cycle, then $\ell(G) \geq 7$.

Proof. Let $C$ be the 6-cycle $\left(v_{0}, v_{1}, v_{2}, v_{3}, v_{4}, v_{5}, v_{0}\right)$ in $G$. Note that each $v_{i}$ has a neighbour $w_{i}$ not in $C$. For $0 \leq i \leq 5$, let $e_{i}$ be the edge $v_{i} v_{i+1}$ (all indices being read modulo 6 ). Let $G^{\prime}$ be the graph obtained from $G-\left\{e_{1}, e_{3}, e_{5}\right\}$ by suppressing the 6 vertices in $C$ which are now of degree 2. By Lemma 3.7.2, $G^{\prime}$ is 2-connected and cubic, in particular, $G^{\prime} \neq \mathbb{P}^{*}$. As $G$ is cubic and $|V(G)| \geq 11$, we see that $|V(G)| \geq 12$ and $\left|V\left(G^{\prime}\right)\right|=|V(G)|-6 \geq 6$. It is important to note that, since $G$ has no 3- or 4-cycles, if $i$ and $j$ are distinct and $w_{i}=w_{j}$, 
then $j=i+3$. Thus, the three new edges $w_{0} w_{1}, w_{2} w_{3}, w_{4} w_{5}$ can't all be incident with the same vertex in $G^{\prime}$.

If $\ell\left(G^{\prime}\right) \geq 7$, then let $F^{\prime}$ be a 2-factor of $G^{\prime}$ having a cycle of length $\ell\left(G^{\prime}\right)$. If $\ell\left(G^{\prime}\right) \leq$ 6 , then $G^{\prime}$ is one of $K_{3,3}, K_{3} \square K_{2}$ or $\mathbb{P}$. When $G^{\prime}$ is $\mathbb{P}, F^{\prime}$ may be chosen to contain exactly one or two new edges, and no more than one in each component. Since $w_{0} w_{1}, w_{2} w_{3}, w_{4} w_{5}$ don't have a common vertex, when $G^{\prime}=K_{3,3}$, we can let $F^{\prime}$ be a 6-cycle containing all three of them.

When $G^{\prime}=K_{3} \square K_{2}$ we observe that, since $G$ is triangle-free, each of the triangles in $G^{\prime}$ must contain one of the new edges. However, no triangle can contain two new edges, say $w_{0} w_{1}$ and $w_{2} w_{3}$, since then $w_{0}=w_{3}$, making $w_{1}$ and $w_{2}$ adjacent. Up to symmetry, there are two ways to pick one edge from each triangle. In both cases, there is (up to symmetry) only one way to choose the third edge not from the triangles to also eliminate all 4-cycles. In either case, we can let $F^{\prime}$ be a 6-cycle containing all 3 new edges.

Expanding $F^{\prime}$ back to $G$, we obtain a partial 2-factor $F$ of $G$ with respect to $C$. Set $k=|E(C) \cap E(F)|$ and let $s$ be the number of vertices in the subgraph induced by $C$ and the cycles in $F$ that meet $C$. If $\left|V\left(G^{\prime}\right)\right|=6$, then $|V(C)|=6=2 k$ and $F$ consists of one cycle, so $\ell(G) \geq \ell(F)=12$. If $G^{\prime}=\mathbb{P}$, then either $k=1$ and $s=6+5$, or $k=2$ and $s=6+10$, and in either case $\ell(G) \geq 8$. If $\ell\left(G^{\prime}\right) \geq 7$, then, by Lemma 3.8, $\ell(G) \geq \ell(F) \geq \ell\left(F^{\prime}\right) \geq 7$, unless $1 \leq k \leq 2$ and a longest cycle $C^{\prime}$ of $F^{\prime}$ contains a new edge. Since $s \geq|V(C)|+\left|V\left(C^{\prime}\right)\right| \geq 6+7$, we get $\ell(G) \geq 13 / k>6$, as desired.

The next two claims will now complete the proof.

Claim 7. If $G$ has a vertex of degree 2 , then $G=\mathbb{P}^{*}$ or $\ell(G) \geq 7$.

Proof. If the vertex $t$ of degree 2 in $G$ is not in a 5- or 6-cycle in $G$, then any 2-factor $F$ of $G$ suffices, as the cycle of $F$ through $t$ is long. If $t$ is in a 6-cycle, then Claim 5 shows either $G=\mathbb{P}^{*}$ or $\ell(G) \geq 7$. The remaining case is that $t$ is in a 5 -cycle $C=$ $\left(v_{0}, v_{1}, v_{2}, v_{3}, v_{4}, v_{0}\right)$, with the labelling chosen so that $v_{0}=t$. For $1 \leq i \leq 4$, let $w_{i}$ be the neighbour of $v_{i}$ not in $C$. As $G$ has no 3- or 4-cycles, these four $w_{i}$ are distinct.

Contracting $v_{0} v_{1}$ to apply Lemma 3.6.3, we deduce that the graph $\left(G-v_{0}\right)-v_{2} v_{3}$ is 2-connected. As $G$ has no 4-cycles, suppressing the four vertices $v_{1}, v_{2}, v_{3}, v_{4}$ of degree 2 in $\left(G-v_{0}\right)-v_{2} v_{3}$ results in a graph $G^{\prime}$ with new edges $w_{1} w_{2}$ and $w_{3} w_{4}$. Obviously, $G^{\prime}$ is 2-connected and cubic and $\left|V\left(G^{\prime}\right)\right|=|V(G)|-5 \geq 6$. If $G^{\prime}=K_{3,3}$, then $G^{\prime}$ contains a 4-cycle avoiding the new edges, which is thus a 4-cycle in $G$, a contradiction. Similarly, if $G^{\prime}=K_{3} \square K_{2}$, then $G^{\prime}$ contains either a 3-cycle or a 4-cycle without a new edge, a contradiction. Thus $\left|V\left(G^{\prime}\right)\right| \geq 8$, so by induction $G^{\prime}=\mathbb{P}$ or $\ell\left(G^{\prime}\right) \geq 7$.

If $G^{\prime}$ is $\mathbb{P}$, then it has a 2 -factor $F^{\prime}$ that contains exactly one of the two new edges $w_{1} w_{2}$ and $w_{3} w_{4}$. If $\ell\left(G^{\prime}\right) \geq 7$, we let $F^{\prime}$ be any longest 2-factor of $G^{\prime}$.

Expanding $F^{\prime}$ back to $G$, we obtain a partial 2-factor $F$ of $G$ with respect to $C$. Set $k=|E(C) \cap E(F)|$ and let $s$ be the number of vertices in the subgraph induced by $C$ and the cycles in $F$ that meet $C$. Then $k=1$ when $G^{\prime}=\mathbb{P}$. By Lemma 3.8, $\ell(G) \geq$ $\ell(F) \geq \ell\left(F^{\prime}\right) \geq 7$, unless $1 \leq k \leq 2$ and a longest cycle $C^{\prime}$ of $F^{\prime}$ contains a new edge. Since $s \geq|V(C)|+\left|V\left(C^{\prime}\right)\right|$ it follows that for $\ell\left(G^{\prime}\right) \geq 7$ we obtain $\ell(G) \geq 12 / k \geq 6$, but observe that equality can only hold if $k=2$ and $V(C) \cup V\left(C^{\prime}\right)$ yields two 6-cycles. One of these 6-cycles contains $v_{0}=t$, and the result follows by Claim 5. If $G^{\prime}=\mathbb{P}$, then $\ell(G) \geq(5+5) / 1=10$. 
Claim 8. If $G$ is cubic, then $\ell(G) \geq 7$.

Proof. Again, we may assume $G$ is cyclically 4-connected and has no 3- or 4-cycles. Claim 6 allows us to assume that $G$ has no 6-cycles. We may further assume $\left(v_{0}, v_{1}, v_{2}, v_{3}\right.$, $\left.v_{4}, v_{0}\right)$ is a 5-cycle $C$ in $G$, as otherwise we are done: every 2-factor has a long cycle.

Let $w_{i}$ be the neighbour of $v_{i}$ that is not in $C$, and let $W=\left\{w_{0}, w_{1}, w_{2}, w_{3}, w_{4}\right\}$. As $G$ has no 3 - or 4-cycles, the vertices $v_{0}, v_{1}, \ldots, v_{4}, w_{0}, w_{1}, \ldots, w_{4}$ are distinct. Furthermore, the vertices in $W$ are pairwise non-adjacent, since $G$ has no 4- or 6-cycles.

Now let $G^{\prime}$ be obtained from $\left(G-v_{0}\right)-v_{2} v_{3}$ by suppressing the four vertices $v_{1}, v_{2}$, $v_{3}, v_{4}$ of degree 2. By Lemma 3.7.1, $G^{\prime}$ is a 2-connected near-cubic graph with degree 2 vertex $w_{0}$. Clearly $\left|V\left(G^{\prime}\right)\right| \geq 6$, so by induction either $G^{\prime}=\mathbb{P}^{*}$ or $\ell\left(G^{\prime}\right) \geq 7$. There are four different 6 -cycles through $w_{0}$ in $\mathbb{P}^{*}$; no set of two edges not incident with either $w_{0}$ or its neighbors meets all four of these. Thus, if $G^{\prime}=\mathbb{P}^{*}$, then we can find a 6-cycle of $G^{\prime}$ containing $w_{0}$ that avoids $w_{1} w_{2}$ and $w_{3} w_{4}$. This is a 6-cycle in $G$, contradicting the fact that $G$ has no 6-cycles. Therefore $G^{\prime} \neq \mathbb{P}^{*}$, and it remains to consider $\ell\left(G^{\prime}\right) \geq 7$.

Let $F^{\prime}$ be a longest 2-factor of $G^{\prime}$. Expanding $F^{\prime}$ back to $G$, we obtain a partial 2factor $F$ of $G$ with respect to $C$. Set $k=|E(C) \cap E(F)|$ and let $s$ be the number of vertices in the subgraph induced by $C$ and the cycles in $F$ that meet $C$. By Lemma 3.8, $\ell(G) \geq \ell(F) \geq \ell\left(F^{\prime}\right) \geq 7$, unless $1 \leq k \leq 2$ and a longest cycle $C^{\prime}$ of $F^{\prime}$ contains a new edge. Since $s \geq|V(C)|+\left|V\left(C^{\prime}\right)\right| \geq 5+7$ we obtain $\ell(G) \geq 12 / k \geq 6$, but observe that equality can only hold if $G$ contains a 6 -cycle, in which case Claim 6 finishes the proof. $\triangle$

Obviously Theorem 3.1 follows from Claims 7 and 8 .

\section{Infinite Graphs}

In this section, we indicate the connections to 2-factors in infinite cubic graphs. The graphs $J(k)$ from Section 2 have a natural limit version $J(\infty)$ (let $k$ go to infinity). This graph is 3 -connected, 1 -ended, and cubic and every 2-factor in $J(\infty)$ contains only cycles of length at most 16 .

In a slightly different direction, we show how to construct a 3-connected, 1-ended, cubic graph so that every 2-factor is a 2-way infinite Hamilton path. The construction starts by noting that the edges of $\mathbb{P}$ partitions into five sets of three edges each with the property that every 2-factor of $\mathbb{P}$ contains precisely two edges from each of the five sets, and these two edges are in different components of the 2-factor.

Let $\mathbb{P}^{+}$be the 3 -connected cubic graph obtained from $\mathbb{P}$ by subdividing the three edges in one of the five sets and then adding a new vertex $n$ adjacent to the three vertices of subdivision. Let $u$ be any vertex of $\mathbb{P}$ not incident with any of the three original edges.

The key properties of $\mathbb{P}^{+}$, easily proved from properties of $\mathbb{P}$, are: its only 2 -factors are Hamilton cycles; and $\mathbb{P}^{+}-n$ has no 2 -factor.

Let $G_{1}, G_{2}, \ldots$ be disjoint copies of $\mathbb{P}^{+}$, with $n_{i}$ and $u_{i}$ being the copies of $n$ and $u$, respectively, in $G_{i}$. Iteratively 3-merge the $G_{i}$, starting with $G_{1}$ at $n_{1}$ and $G_{2}$ at $u_{2}$. Then this new graph is 3-merged, at $n_{2}$, with $G_{3}$ at $u_{3}$, and so on. The infinite graph thus created is easily seen to have 2-way infinite Hamilton paths as its only 2-factors.

\section{Open Questions}

Several open questions naturally arise. We mention only a few here. 
Naturally, we wonder what the exact values of $L_{2}(n)$ and $L_{3}(n)$ are and we conjecture that $L_{k}(n)$ is unbounded for sufficiently large $k$. This can be viewed as a weaker version of the following unpublished question Thomassen first asked in the 1970's: Is every graph in $\mathcal{C}_{k}(n)$ Hamiltonian when $k$ is sufficiently large?

Kochol (personal communication, based on [4, 5]) has examples of cyclically 6-connected cubic graphs on $2 n$ vertices for which every 2 -factor has $c n$ components, for some $c>0$. Of course this does not imply that $L_{6}(n)$ is bounded.

On the other hand, Fleischner [1] conjectures that every cyclically 4-connected Class 2 graph has a dominating cycle. This might suggest that there is always a 2-factor with a long cycle for cyclically 4-connected cubic graphs.

Two other natural questions are: what is the average value of $\ell(G)$ over all graphs in either $C_{2}(n)$ or $C_{3}(n)$ ? and what is $\ell(R)$, where $R$ is the random cubic graph?

\section{Acknowledgements}

We would like to thank Ron Gould, Martin Kochol and Carsten Thomassen for useful discussions regarding this paper. We are also grateful to the referees for their helpful comments.

\section{References}

[1] H. Fleischner, Cycle decompositions, 2-coverings, removable cycles, and the four-color-disease, Progress in graph theory, Waterloo, Ont., 1982, 233-246, Academic Press, Toronto, ON, 1984.

[2] D. A. Holton and J. Sheehan, The Petersen Graph, Australian Mathematical Society Lecture Series, 7, Cambridge University Press, Cambridge, 1993.

[3] B. Jackson and K. Yoshimoto, Spanning even subgraphs of 3-edge-connected graphs, J. Graph Theory 62 (2009), 37-47.

[4] M. Kochol, Superposition and constructions of graphs without nowhere-zero k-flows, Europ. J. Combin. 23 (2002), 281-306.

[5] M. Kochol, Equivalences between hamiltonicity and flow conjectures, and the sublinear defect property, Discrete Math. 254 (2002), 221-230.

[6] J. Petersen, Die Theorie der regulären Graphen, Acta Math. 15 (1891), 193-220.

[7] T. Schönberger, Ein Beweis des Petersenschen Graphensatzes, Acta Scientia Mathematica Szeged 7 (1934), 51-57.

[8] W. T. Tutte, The factors of graphs, Canad. J. Math. 4 (1952), 314-328.

[9] D. B. West, Introduction to Graph Theory, 2nd ed., Prentice-Hall, Upper Saddle River, NJ, 2001. 